\title{
Density Evolution for SUDOKU codes on the Erasure Channel
}

\author{
Caroline Atkins and Jossy Sayir \\ University of Cambridge
}

\begin{abstract}
Codes based on SUDOKU puzzles are discussed, and belief propagation decoding introduced for the erasure channel. Despite the non-linearity of the code constraints, it is argued that density evolution can be used to analyse code performance due to the invariance of the code under alphabet permutation. The belief propagation decoder for erasure channels operates by exchanging messages containing sets of possible values. Accordingly, density evolution tracks the probability mass functions of the set cardinalities. The equations governing the mapping of those probability mass functions are derived and calculated for variable and constraint nodes, and decoding thresholds are computed for long SUDOKU codes with random interleavers.
\end{abstract}

\section{INTRODUCTION}

The analogy between SUDOKU puzzles and low-density parity-check (LDPC) has been widely noted ([1], [2], [3]). Both can be represented by a factor graph, where the constraints for LDPC codes are linear, i.e., $\sum c_{i} x_{i}=0$ where the coefficients and sum are defined over a finite field, while for SUDOKU the constraints are non linear, requiring all variable in a constraint to have different values within a finite alphabet. This analogy can be pursued further by investigating the code defined by the set of valid SUDOKU grids and its properties with respect to communication over noisy channels. This investigation brings up a number of interesting questions, some of which we have been able to provide answers for, while others remain unsolved:

- what is the rate of a code defined as the set of valid (solved) SUDOKU grids? (unsolved for general dimensions)

- how are SUDOKU encoded, e.g., how can we map information sequences to the set of valid SUDOKU grids (some solutions proposed, not covered in the present paper)

- can SUDOKU codes be decoded via Belief Propagation (BP) for general "soft" channels? (solved in [2], covered here)

- error performance of SUDOKU codes (unsolved in general)

- BP for the erasure channel? (solutions covered here)

- performance of BP for the erasure channel (covered here).

In the present paper, we liberate SUDOKU puzzles from their strict definition on a $q \times q$ grid with row, column and subgrid

Funded in part by the European Research Council under ERC grant agreement 259663 and by the FP7 Network of Excellence NEWCOM\# under grant agreement 318306 . constraints, and allow puzzles to grow to any length, with each variable involved in a numbder $d_{v}$ of constraints, and each constraint involving a number $d_{c}$ of variables. This is similar to regular long LDPC codes. The aim of the paper is to provide analysis of the performance of long SUDOKU codes using density evolution, a technique originally developed to analyse LDPC codes. As we will see, this is slightly more difficult that one could expect because SUDOKU codes are non-linear and hence have no all-zero codeword, and performance analysis for a given transmitted codeword would be insufficient anyhow. It is therefore quite surprising that density evolution can be used after all.

In the next section, we will explain the decoder operations for a SUDOKU code. In Section III, we will develop density evolution for SUDOKU codes, and finally provide results, a conclusion and remaining open problems in the last section.

\section{DECODER}

Belief propagation for non-binary codes over an alphabet of size $q$ normally operates on a factor graph by exchanging messages in the form of $q$-ary a-posteriori probability distributions for the $q$-ary code symbols. This is also true of SUDOKU codes, and the node operations for general channels can easily be derived using Bayesian inference. We present these mappings here without further discussion as

$$
q_{i j}=\alpha_{i} \prod_{k \neq i} p_{k j}
$$

for variable nodes, where $p_{k j}$ is the $j$-th entry of the $k$-th probability-valued incoming message to the variable node, while $\alpha_{i}$ is the a-posteriori probability that the variable takes on the $i$-th value given the channel observation. For constraint nodes of degree equal to the alphabet size $q$, the mapping is

$$
q_{i j}=\xi_{i} \sum_{\left(j_{1}, \ldots, j_{i}=j, \ldots, j_{q}\right) \in S_{q}} \prod_{k \neq i} p_{k j_{k}} .
$$

where $\xi_{i}$ is a normalisation constant and $S_{q}$ is the symmetric group on $\{1,2, \ldots, q\}$. This can also be written as

$$
q_{i j}=\frac{p_{i j}}{\operatorname{perm}(P)} \operatorname{perm}\left(P_{i j}\right) .
$$

where $\operatorname{perm}(A)$ is the Cauchy permanent of a matrix $A, P$ is the $q \times q$ matrix of incoming messages, and $A_{i j}$ for any matrix $A$ denotes the matrix obtained by removing the $i$-th row and 
$j$-th column from $A .^{1}$ These rules can readily be extended to constraint nodes of any positive degree $d_{c} \leq q$, where all variables in a constraint must take on different values (they cover all values in $\{1, \ldots, q\}$ only when $d_{c}=q$ ).

A $q$-ary erasure channel with input variable $X$ defined over the alphabet $\mathcal{X}$ of cardinality $q$, and output alphabet $Y$ defined over $\mathcal{X} \cup\{\varepsilon\}$ where $\varepsilon$ is the erasure symbol, has transition probabilities

$$
\begin{cases}P_{Y \mid X}(y \mid x)=1-\delta & \text { for } y=x, \text { and } \\ P_{Y \mid X}(y \mid x)=\delta & \text { for } y=\varepsilon .\end{cases}
$$

Note that the corresponding a-posteriori distributions are $P_{X \mid Y}(x \mid \varepsilon)=1 / q$ for all $x$, and $P_{X \mid Y}(x \mid y)$ is 1 if $x=y$ and 0 otherwise. Hence, for an erasure channel, messages will start off as either atomic distributions assigning a probability of 1 to the observed symbol and 0 to all others, or uniform distributions on the complete alphabet. Node operations will only ever yield uniform distributions, where these would normally narrow down to a subset of the alphabet as certain symbols get excluded, until all messages converge to atomic distribution in the event that decoding is successful. Therefore, the message-passing algorithm using distributions can be replaced by a message-passing algorithm where messages are alphabet subsets, where a subset message is equivalent to a distribution message with a uniform distribution over the subset.

Let $m_{c h}$ be an incoming subset message to a variable node from the channel, where $m_{c h}=\{i\}$ if the channel output is symbol $i$, and $m_{c h}=\{1, \ldots, q\}$ if the channel output is an erasure. Let $m_{c \rightarrow v}(k)$ represents the incoming subset message to the same variable node along its $k$-th edge, where $m_{c \rightarrow v}(k) \subseteq\{1, \ldots, q\}$. Translating (1) from distributions to subset notation yields, for the $k$-th outgoing message $m_{v \rightarrow c}(k)$ of the variable node

$$
m_{v \rightarrow c}(k)=m_{c h} \cap \bigcap_{k^{\prime} \neq k} m_{c \rightarrow v}\left(k^{\prime}\right),
$$

i.e., the outgoing message is an extrinsic intersection of all incoming messages, since any zeros in an incoming distribution would eliminate all non-zero probabilities for the same symbol in other incoming distributions.

For constraint nodes, the relation between the distribution message operation (2) and the subset operation is slightly more intricate. The output $q_{i j}$ is zero if there exists a restriction of the matrix $P$ of incoming messages to the set of rows $\mathcal{I}^{\prime}=\left\{i_{1}^{\prime}, \ldots, i_{k}^{\prime}\right\}$ not including $i$ whose non-zero entries form a square matrix over the set of columns $\mathcal{J}^{\prime}=\left\{j_{1}^{\prime}, \ldots, j_{k}^{\prime}\right\}$ including $j$. This square sub-matrix constitutes a bottleneck in the matrix $P$ so that every non-zero product of elements $\prod_{k} p_{k j_{k}}$ for a permutation $\left(j_{1}, \ldots, j_{q}\right) \in S_{q}$ must pass through

${ }^{1}$ Note that, while the relation between SUDOKU constraint node operations and the Cauchy permanent was never formally established in [3]. it is the reason why Sinkhorn can be used to solve SUDOKU puzzles. Sinkhorn will converge towards a doubly stochastic output matrix, and (3) makes it evident that the Bayesian optimal output message matrix of a constraint node is doubly stochastic. the bottleneck, i.e., $k \in \mathcal{I}^{\prime} \Longrightarrow j_{k} \in \mathcal{J}^{\prime}$. Therefore all of the terms in the expression (2) for $q_{i j}$ are zero when $i \notin \mathcal{I}^{\prime}$ and $j \in \mathcal{J}^{\prime}$. Translated in terms of subset messages, square submatrices occur whenever the union of $k$ incoming subset messages has cardinality $k$. Letting $m_{v \rightarrow c}(i) \subseteq\{1, \ldots, q\}$ be the incoming subset message to a constraint node on its $i$-th edge, the resulting constraint node rule for generating the $j$-th outgoing message $m_{c \rightarrow v}(j) \subseteq\{1, \ldots, q\}$ is

$$
m_{c \rightarrow v}(j)=\{1, \ldots, q\}-\bigcup_{n} A_{n}
$$

where $A_{n}$ is any set such that

$$
\exists \mathcal{J} \subset\{1, \ldots, q\} \text { such that }\left\{\begin{array}{l}
k \notin \mathcal{J}, \\
A_{n}=\bigcup_{j \in \mathcal{J}} m_{v \rightarrow c}(j), \\
\text { and } \# \mathcal{J}=\# A_{n},
\end{array}\right.
$$

where $\# S$ denotes the cardinality of the set $S$.

It is worth noting that the subset operations just stated are familiar to passionate Sudoku solvers and are described in Sudoku solving guides as the basic rules for solving most puzzles by logic excluding the fiendishly difficult ones that require constraint combination tricks or guessing. The constraint rule above with $\# A_{n}=1$ correspond to the simple rule of eliminating any candidate value for a cell that is already used by another cell in the same constraint. The same rule for $\# A_{n}=2$ corresponds to eliminating pairs of values that are shared among two other cells in the same constraint, and so forth. Hence, we would claim that most people who enjoy solving Sudoku puzzles are in fact running a belief propagation algorithm for erasure channels in their brains.

\section{DEnsity Evolution FOR SUDOKU CODES}

Density evolution ([4]) for LDPC codes relies on a number of properties that generalise to the codes with SUDOKUtype constraints considered here. In particular, concentration and convergence to the cycle-free (tree-like) case apply to any code with local constraints that can be represented as a factor graph, and this is the case for the SUDOKU-type codes. However, one of the crucial properties that simplifies density evolution for LDPC codes does not generalise to SUDOKU codes: the sufficiency of the analysis given the allone codeword. Indeed, the all-one sequence is no longer a codeword for the SUDOKU case. Indeed, even for optimal maximum likelihood (ML) decoding, let alone sub-optimal iterative decoding, performance analysis given a specific codeword does not suffice in general for non-linear codes, as the weight-distance equivalence property of linear codes does not extend to non-linear codes.

In principle, in order to make binding statements about the performance of iterative decoding for SUDOKU codes, one would need to compute separate density evolution recursions for every possible pattern of transmitted code symbols. However, the following lemma listing the symmetries that all node operations fulfills and allows us to overcome this hurdle:

Lemma 1: Consider a node of degree $d$ in a factor graph and consider an extrinsic mapping from any $d-1$ of its input 
messages to the remaining output message, when decoding for the $q$-ary erasure channel. This mapping is invariant under the following:

- any of the $(d-1)$ ! re-orderings of its input messages

- any of the $q$ ! permutations of the code alphabet and corresponding re-shuffling of the subset-valued intput and output messages

The lemma allows us to make the following simplifying assumptions when computing the density evolution recursion:

- for a variable node, assume that the transmitted variable has value 1 . Due to the nature of the erasure decoder, this implies that the subset-valued messages from the channel and in- and outcoming along all edges to a variable node will contain at least the value 1 .

- for a constraint node, assume that the transmitted variables corresponding to input edges 1 to $d-1$ have values 1 to $d-1$ respectively, and the transmitted variable corresponding to the output message has value $d$.

Despite the simplifications following from Lemma 1, the message alphabet for density evolution is still rather large. The following proposition that also follows from Lemma 1 enables us to operate density evolution on a considerably reduced message alphabet:

Proposition 1: For performance analysis, the probability distribution of the cardinalities of subset-valued messages is a sufficient statistic for the probability distribution of the messages themselves.

Hence, it will be sufficient to track the cardinality of messages, assuming for variable nodes that each message contains at least the value 1 , and for constraint nodes that incoming messages contain the values 1 to $d-1$, respectively, and the output message the value $d$. The cardinalities have value between 1 and $q$ (value zero corresponding to the empty subset can never occur for an erasure channel as the channel makes no mistake.)

The actual mappings of cardinalities are non-trivial and still subject to a combinatorial explosion with growing $q$ and $d$. We will discuss the computation of these mappings in the next two subsections, and then present results for $q=d$ and $q$ between 3 and 6. Note that we currently have the computing power to go up to $q=8$ and hope to find further efficient implementation that would allow us to push the boundary to $q=9$, but this is the limit beyond which the combinatorial explosion of terms in the density evolution recursion would probably be beyond anyone's computational abilities.

\section{A. Variable Node Equations}

Consider a variable node of degree $d_{v}$ operating over an alphabet of size $q$. We now consider the extrinsic mapping of messages from $d_{v}-1$ inputs to one output. We are assuming incoming messages and hence their cardinalities to be independent and have identical distributions of cardinality $P_{V i}(k)$ for $k=1, \ldots, q$. Note that $P_{V i}(0)=0$ because any message always contains at least the corresponding transmitted symbol for an erasure channel (the channel makes no "errors").

Density evolution aims to express the probability distribution of the cardinality of the output message $P_{V o}($.$) in function$ of $P_{V i}($.$) . In line with the assumptions above, we can assume$ without loss of generality that the true value of the variable is 1 and hence all input messages and the output messages must necessarily contain a 1 .

The density evolution analysis of a variable node is best first illustrated with an example. Take $q=4, d_{v}=3$. In order to help avoid confusion, we will use bold numbers, e.g., 1.2, ... to denote set cardinalities and normal numbers, e.g., $1,2, \ldots$ to denote possible values of variables in a SUDOKU code. There are 16 combinations of cardinalities for the two input messages, i.e., $(\mathbf{1}, \mathbf{1}),(\mathbf{1}, \mathbf{2}), \ldots,(\mathbf{2}, \mathbf{1}), \ldots$. Clearly we can restrict our attention to non-decreasing combinations since the two input edges are essentially interchangeable. The number of non-decreasing combinations for general $q$ and $d_{v}-1$, which we call $\mathcal{N}\left(q, d_{v}-1\right)$, can be defined recursively as

$$
\left\{\begin{array}{l}
\mathcal{N}(a, b)=\sum_{k=1}^{a} \mathcal{N}(k, b-1) \\
\mathcal{N}(a, 1)=a
\end{array}\right.
$$

In our example, this gives $\mathcal{N}(4,2)=10$ as can easily be verified by listing them: $(\mathbf{1}, \mathbf{1}),(\mathbf{1}, \mathbf{2}),(\mathbf{1}, \mathbf{3}),(\mathbf{1}, \mathbf{4})$, $(2,2),(2,3),(2,4),(3,3),(3,4)$, and $(4,4)$. Let us take the combination $(\mathbf{2}, \mathbf{3})$ as an example. Since both input messages must contain a 1 , this implies a uniform distribution over the sets $\{1,2\},\{1,3\}$, and $\{1,4\}$ for the message of cardinality 2 , and a uniform distribution over the messages $\{1,2,3\},\{1,2,4\}$ and $\{1,3,4\}$ for the message of cadinality 3. There are 9 possible combinations of those, 3 of which will yield the output message $\{1\}$ of cardinality 1 , and 6 of which will yield an output message of cardinality 2 , either $\{1,2\},\{1,3\}$ or $\{1,4\}$. These probabilities can be added to obtain the overall distribution of output cardinalities, taking care to multiply the resulting probabilities by the number of combinations, e.g., the combination of cardinalities $(\mathbf{2}, \mathbf{3})$ counts double for $(\mathbf{2}, \mathbf{3})$ or $(\mathbf{3}, \mathbf{2})$ while the combination of cardinalities $(\mathbf{2}, \mathbf{2})$ counts only for itself. This process is best illustrated with tables. Table I shows the probabilities of output cardinalities given combinations of input cardinalities and the corresponding multiplicity factor The entries in the non-

\begin{tabular}{|c|c||c|c|c|c|}
\hline & \multicolumn{1}{|c||}{} & \multicolumn{5}{c|}{ Output \# } \\
input \# & multipl. & $\mathbf{1}$ & $\mathbf{2}$ & $\mathbf{3}$ & $\mathbf{4}$ \\
\hline \hline$(\mathbf{1}, \mathbf{1})$ & 1 & 1 & 0 & 0 & 0 \\
\hline$(\mathbf{1}, \mathbf{2})$ & 2 & 1 & 0 & 0 & 0 \\
\hline$(\mathbf{1}, \mathbf{3})$ & 2 & 1 & 0 & 0 & 0 \\
\hline$(\mathbf{1}, \mathbf{4})$ & 2 & 1 & 0 & 0 & 0 \\
\hline$(\mathbf{2}, \mathbf{2})$ & 1 & $2 / 3$ & $1 / 3$ & 0 & 0 \\
\hline$(\mathbf{2}, \mathbf{3})$ & 2 & $1 / 3$ & $2 / 3$ & 0 & 0 \\
\hline$(\mathbf{2}, \mathbf{4})$ & 2 & 0 & 1 & 0 & 0 \\
\hline$(\mathbf{3}, \mathbf{3})$ & 1 & 0 & $2 / 3$ & $1 / 3$ & 0 \\
\hline$(\mathbf{3}, \mathbf{4})$ & 2 & 0 & 0 & 1 & 0 \\
\hline$(\mathbf{4}, \mathbf{4})$ & 1 & 0 & 0 & 0 & 1 \\
\hline
\end{tabular}

TABLE I

OUTPUT PROBABILITIES AND MULTIPLICITIES FOR $q=4$ AND $d_{v}=3$

trivial columns in Table I corresponding to input configurations $(\mathbf{2}, \mathbf{2}),(\mathbf{2}, \mathbf{3})$ and $(\mathbf{3}, \mathbf{3})$ can be visualised in the following 
tables listing all possible pairs of inputs with these cardinalities and the corresponding output cardinality,

\begin{tabular}{|c||c|c|c|}
\hline$(\mathbf{2}, \mathbf{2})$ & $\{1,2\}$ & $\{1,3\}$ & $\{1,4\}$ \\
\hline \hline$\{1,2\}$ & $\mathbf{2}$ & $\mathbf{1}$ & $\mathbf{1}$ \\
\hline$\{1,3\}$ & $\mathbf{1}$ & $\mathbf{2}$ & $\mathbf{1}$ \\
\hline$\{1,4\}$ & $\mathbf{1}$ & $\mathbf{1}$ & $\mathbf{2}$ \\
\hline
\end{tabular}

\begin{tabular}{|c||c|c|c|}
\hline$(\mathbf{2}, \mathbf{3})$ & $\{1,2,3\}$ & $\{1,2,4\}$ & $\{1,3,4\}$ \\
\hline \hline$\{1,2\}$ & $\mathbf{2}$ & $\mathbf{2}$ & $\mathbf{1}$ \\
\hline$\{1,3\}$ & $\mathbf{2}$ & $\mathbf{1}$ & $\mathbf{2}$ \\
\hline$\{1,4\}$ & $\mathbf{1}$ & $\mathbf{2}$ & $\mathbf{2}$ \\
\hline
\end{tabular}

\begin{tabular}{|c||c|c|c|}
\hline$(\mathbf{3}, \mathbf{3})$ & $\{1,2,3\}$ & $\{1,2,4\}$ & $\{1,3,4\}$ \\
\hline \hline$\{1,2,3\}$ & $\mathbf{3}$ & $\mathbf{2}$ & $\mathbf{2}$ \\
\hline$\{1,2,4\}$ & $\mathbf{2}$ & $\mathbf{3}$ & $\mathbf{2}$ \\
\hline$\{1,3,4\}$ & $\mathbf{2}$ & $\mathbf{2}$ & $\mathbf{3}$ \\
\hline
\end{tabular}

Finally, the resulting distribution of output cardinalities can be read out directly from Table I to yield

$$
\begin{aligned}
P_{v o}(\mathbf{1})= & \left(P_{v i}(\mathbf{1})\right)^{2}+2 P_{v i}(\mathbf{1})\left(P_{v i}(\mathbf{2})+P_{v i}(\mathbf{3})+P_{v i}(\mathbf{4})\right) \\
& +\frac{2}{3}\left(P_{v i}(\mathbf{2})\right)^{2}+\frac{2}{3} P_{v i}(\mathbf{2}) P_{v i}(\mathbf{3}) \\
P_{v o}(\mathbf{2})= & \frac{1}{3}\left(P_{v i}(\mathbf{2})\right)^{2}+\frac{4}{3} P_{v i}(\mathbf{2}) P_{v i}(\mathbf{3})+2 P_{v i}(\mathbf{2}) P_{v i}(\mathbf{4}) \\
& +\frac{2}{3}\left(P_{v i}(\mathbf{3})\right)^{2} \\
P_{v o}(\mathbf{3})= & \frac{1}{3}\left(P_{v i}(\mathbf{3})\right)^{2}+2 P_{v i}(\mathbf{3}) P_{v i}(\mathbf{4}) \\
P_{v o}(\mathbf{4})= & \left(P_{v i}(\mathbf{4})\right)^{2}
\end{aligned}
$$

The whole process can be summarized and generalized to any $q$ and $d_{v}$ as follows

$$
P_{V o}(k)=\sum_{\mathbf{j}: j_{2} \leq j_{3} \leq \ldots j_{d_{v}}} \Gamma(\mathbf{j}) P_{V o \mid V i_{1} \ldots V i_{d_{v}-1}}\left(k \mid j_{2} \ldots j_{d_{v}}\right)
$$

where

$$
\Gamma\left(x_{1}, \ldots, x_{n}\right)=\frac{n !}{\prod_{i} \#\left\{x_{m}=i\right\} !}
$$

where \# denotes the cardinality of a set. This can be further developed to give

$$
P_{V o}(k)=\sum_{\mathbf{j}: j_{2} \leq \ldots j_{d_{v}}} \Gamma(\mathbf{j}) \frac{\#\left\{m_{v o}: \# m_{v o}=k\right\}}{\#\left\{m_{v o}\right\}} \prod_{m=2}^{d_{v}} P_{V i}\left(j_{m}\right)
$$

where the cardinalities of output message sets in the fraction are sets of possible output messages $m_{v o}$ given all possible input messages of the cardinalities given by $\mathbf{j}=\left(j_{2}, \ldots, j_{d_{v}}\right)$.

\section{B. Constraint Node Equations}

Consider a constraint node of degree $d_{c}$ operating over an alphabet of size $q$. For a classical SUDOKU puzzle, we have $d_{c}=q$, i.e., each constraint ties $q$ variables to a permutations of the numbers 1 to $q$, but we can also consider the more general case where $d_{c} \neq q$ and each constraint requires $d_{c}$ variables to take on distinct values among the numbers 1 to 1 . We consider the mapping of messages from $d_{c}-1$ inputs to one output. We are assuming incoming messages and hence their cardinalities to be independent and have identical distributions of cardinality $P_{c i}(k)$ for $k=1, \ldots, q$. Note that $P_{c i}(0)=0$ because any message always contains at least the corresponding transmitted symbol for an erasure channel (the channel makes no "errors").

We will again aim to express the probability distribution of the cardinality of the output message $P_{c o}($.$) in function of$ $P_{c i}($.$) . In line with the assumptions above, we can assume$ without loss of generality that the oputput message goes to a variable with true value 1 . The output message must therefore contain a 1 . The input messages each come from variables with different true values. The messages must each contain the value held by their source variable. Without loss of generality we can assume these values to be $2, \ldots, d_{c}$.

As for variable nodes in the previous section, the constraint node can again best be demonstrated with an example. Take the case where $q=4$ and $d_{c}=4$. The same conventions are used as with the variable node. There are 64 combinations of input cardinalities. Again these can be grouped in nondecreasing combinations. There are 20 such combinations.

Let us take the combination $(\mathbf{1}, \mathbf{2}, \mathbf{3})$ as an example. The three messages must contain the values of their source node. This implies that the message of cardinality $\mathbf{1}$ is $\{2\}$. There is a uniform distribution over messages $\{1,3\},\{2,3\}$, and $\{3,4\}$ for the message of cardinality $\mathbf{2}$, and a uniform distribution over messages $\{1,2,4\},\{1,3,4\}$, and $\{2,3,4\}$ for the message of cardinality 3 . There are 9 possible combinations of these. Two will yield output message $\{1\}$, these are $\{2\},\{2,3\},\{2,3,4\}$, and $\{2\},\{3,4\},\{2,3,4\}$. Two will yield the output message $\{1,4\}$ of cardinality 2 , these are $\{2\},\{2,3\},\{1,2,4\}$ and $\{2\},\{2,3\},\{1,3,4\}$. The remaining five combinations yield output message $\{1,3,4\}$ of cardinality 3.

\begin{tabular}{|c|c||c|c|c|c|}
\hline & \multicolumn{1}{|c||}{} & \multicolumn{4}{c|}{ output \# } \\
input \# & multipl. & $\mathbf{1}$ & $\mathbf{2}$ & $\mathbf{3}$ & $\mathbf{4}$ \\
\hline \hline$(\mathbf{1}, \mathbf{1}, \mathbf{1})$ & 1 & 1 & 0 & 0 & 0 \\
\hline$(\mathbf{1}, \mathbf{1}, \mathbf{2})$ & 3 & $2 / 3$ & $1 / 3$ & 0 & 0 \\
\hline$(\mathbf{1}, \mathbf{1}, \mathbf{3})$ & 3 & $1 / 3$ & $2 / 3$ & 0 & 0 \\
\hline$(\mathbf{1}, \mathbf{1}, \mathbf{4})$ & 3 & 0 & 1 & 0 & 0 \\
\hline$(\mathbf{1}, \mathbf{2}, \mathbf{2})$ & 3 & $4 / 9$ & $2 / 9$ & $1 / 3$ & 0 \\
\hline$(\mathbf{1}, \mathbf{2}, \mathbf{3})$ & 6 & $2 / 9$ & $2 / 9$ & $5 / 9$ & 0 \\
\hline$(\mathbf{1}, \mathbf{2}, \mathbf{4})$ & 6 & 0 & $1 / 3$ & $2 / 3$ & 0 \\
\hline$(\mathbf{1}, \mathbf{3}, \mathbf{3})$ & 3 & $1 / 9$ & 0 & $8 / 9$ & 0 \\
\hline$(\mathbf{1}, \mathbf{3}, \mathbf{4})$ & 6 & 0 & 0 & 1 & 0 \\
\hline$(\mathbf{1}, \mathbf{4}, \mathbf{4})$ & 3 & 0 & 0 & 1 & 0 \\
\hline$(\mathbf{2}, \mathbf{2}, \mathbf{2})$ & 1 & $8 / 27$ & $1 / 9$ & 0 & $16 / 27$ \\
\hline$(\mathbf{2}, \mathbf{2}, \mathbf{3})$ & 3 & $4 / 27$ & $2 / 27$ & 0 & $21 / 27$ \\
\hline$(\mathbf{2}, \mathbf{2}, \mathbf{4})$ & 3 & 0 & $1 / 9$ & 0 & $8 / 9$ \\
\hline$(\mathbf{2}, \mathbf{3}, \mathbf{3})$ & 3 & $2 / 27$ & 0 & 0 & $25 / 27$ \\
\hline$(\mathbf{2}, \mathbf{3}, \mathbf{4})$ & 6 & 0 & 0 & 0 & 1 \\
\hline$(\mathbf{2}, \mathbf{4}, \mathbf{4})$ & 3 & 0 & 0 & 0 & 1 \\
\hline$(\mathbf{3}, \mathbf{3}, \mathbf{3})$ & 1 & $1 / 27$ & 0 & 0 & $26 / 27$ \\
\hline$(\mathbf{3}, \mathbf{3}, \mathbf{4})$ & 3 & 0 & 0 & 0 & 1 \\
\hline$(\mathbf{3}, \mathbf{4}, \mathbf{4})$ & 3 & 0 & 0 & 0 & 1 \\
\hline$(\mathbf{4}, \mathbf{4}, \mathbf{4})$ & 1 & 0 & 0 & 0 & 1 \\
\hline
\end{tabular}

TABLE II

OUTPUT PROBABILITIES AND MULTIPLICITIES FOR $q=4$ AND $d_{c}=4$ 
Finally, the resulting output cardinalities can be expressed as

$$
\begin{aligned}
& P_{c o}(\mathbf{1})=\left(P_{c i}(\mathbf{1})\right)^{2}+2 P_{c i}(\mathbf{1})^{2} P_{c i}(\mathbf{2})+2 P_{c i}(\mathbf{1})^{2} P_{c i}(\mathbf{3}) \\
&+\frac{4}{3} P_{c i}(\mathbf{1}) P_{c i}(\mathbf{2})^{2}+\frac{4}{3} P_{c i}(\mathbf{1}) P_{c i}(\mathbf{2}) P_{c i}(\mathbf{3}) \\
&+\frac{1}{3} P_{c i}(\mathbf{1}) P_{c i}(\mathbf{3})^{2}+\frac{8}{27} P_{c i}(\mathbf{2})^{3}+\frac{4}{9} P_{c i}(\mathbf{2})^{2} P_{c i}(\mathbf{3}) \\
&+\frac{2}{9} P_{c i}(\mathbf{2}) P_{c i}(\mathbf{3})^{2}+\frac{1}{27} P_{c i}(\mathbf{3})^{3} \\
& P_{c o}(\mathbf{2})= P_{c i}(\mathbf{1})^{2} P_{c i}(\mathbf{2})+2 P_{c i}(\mathbf{1})^{2} P_{c i}(\mathbf{3})+3 P_{c i}(\mathbf{1})^{2} P_{c i}(\mathbf{4}) \\
&+\frac{2}{3} P_{c i}(\mathbf{1}) P_{c i}(\mathbf{2})^{2}+\frac{4}{3} P_{c i}(\mathbf{1}) P_{c i}(\mathbf{2}) P_{c i}(\mathbf{3}) \\
&+2 P_{c i}(\mathbf{1}) P_{c i}(\mathbf{2}) P_{c i}(\mathbf{4})+\frac{1}{9} P_{c i}(\mathbf{2})^{3} \\
&+\frac{2}{9} P_{c i}(\mathbf{2})^{2} P_{c i}(\mathbf{3})+\frac{1}{3} P_{c i}(\mathbf{2})^{2} P_{c i}(\mathbf{4}) \\
& P_{c o}(\mathbf{3})= P_{c i}(\mathbf{1}) P_{c i}(\mathbf{2})^{2}+\frac{10}{3} P_{c i}(\mathbf{1}) P_{c i}(\mathbf{2}) P_{c i}() \\
&+4 P_{c i}(\mathbf{1}) P_{c i}(\mathbf{2}) P_{c i}(\mathbf{4})+\frac{8}{3} P_{c i}(\mathbf{1}) P_{c i}(\mathbf{3})^{2} \\
&+6 P_{c i}(\mathbf{1}) P_{c i}(\mathbf{3}) P_{c i}(\mathbf{4})+3 P_{c i}(\mathbf{1}) P_{c i}(\mathbf{4})^{2} \\
& 16 \\
&+3 P_{c i}(\mathbf{2})^{3}+\frac{7}{3} P_{c i}(\mathbf{2})^{2} P_{c i}(\mathbf{3})+\frac{8}{3} P_{c i}(\mathbf{2})^{2} P_{c i}(\mathbf{4}) \\
&+\frac{25}{9} P_{c i}(\mathbf{2}) P_{c i}(\mathbf{3})^{2}+6 P_{c i}(\mathbf{2}) P_{c i}(\mathbf{3}) P_{c i}(\mathbf{4}) \\
& 3 P_{c i}(\mathbf{2}) P_{c i}(\mathbf{4})^{2}+\frac{26}{27} P_{c i}(\mathbf{3})^{3} \\
& P_{c o}(\mathbf{4})(\mathbf{3}) P_{c i}(\mathbf{4})^{2} P_{c i}(\mathbf{4})^{3} . \\
&
\end{aligned}
$$

\section{Results and Discussion}

The density evolution recursions outlined above can be used to compute thresholds for long SUDOKU-type codes in a similar fashion as is done for LDPC codes. Convergence to cardinality 1 message is the equivalent to error-free decoding, and the threshold is the limit between error-free decoding and values of the erasure probability for which the decoding error after any number of iterations remains positively lower bounded.

However, one major difficulty for SUDOKU codes is that the code rate is unknown and determining it remains an open problem. We do have a conjecture but are unable to give it a full justification at this point: we obtain a rate estimate by counting the number of possible values that a set of variables can take on when those variables are arranged in the tree resulting from considering the decoding neighbourhood of one node in a finite number of iterations within the bi-partite factor graph corresponding to a SUDOKU code. For a regular $\left(d_{v}, d_{c}\right)$ SUDOKU code of alphabet size $q$, this results after $k$ iterations, in a rate

$$
R_{k}=\frac{\log _{q}\left(d_{c} !\left(\left(d_{c}-1\right) !\right)^{k\left(d_{v}-1\right)}\right)}{d_{c}+k\left(d_{c}-1\right)\left(d_{v}-1\right)}
$$

which, as $k$ grows large, tends towards

$$
R=\frac{\log _{q}\left(\left(d_{c}-1\right) !\right)}{d_{c}-1} .
$$

\begin{tabular}{|c|c|c||c|c|}
\hline$q$ & $d_{v}$ & $d_{c}$ & $\theta_{d e}$ & $R$ \\
\hline \hline 3 & 3 & 3 & 0.98426 & 0.3155 \\
\hline 4 & 3 & 4 & 0.94142 & 0.4308 \\
\hline 5 & 3 & 5 & 0.89843 & 0.4937 \\
\hline 6 & 3 & 6 & 0.86026 & 0.5344 \\
\hline
\end{tabular}

TABLE III

THRESHOLD VS. CONJECTURED RATE FOR LONG SUDOKU CODES

It is somewhat surprising that our final conjectured rate for long codes does not depend on the variable degree of the nodes but only on the constraint node degree $d_{c}$ and on the alphabet size $q$. This may indicate a weakness of our construction but more analysis is needed to fully understand whether the number we calculate here is close to the actual rate or merely an upper bound.

In Table III, we list the thresholds (in terms or erasure probability) calculated through densitye evolution and the conjectured estimated rates resulting from (4). The table indicates that there is a wide gap between threshold and rate at this point, where the gap appears to become thinner as the alphabet size grows. However, it should be noted that the rate estimate is only a conjecture at this point assuming that the whole inifite length codeword can be represented as a tree-like decoding neighbourhood. More theory is needed to test this assumption. Intuitively, we expect the rates currently provided to be upper bounds on the true rate.

\section{CONCLUSION}

We have presented the essential components of an erasure iterative decoder for locally decodable codes fulfilling SUDOKU-type constraints. We have shown that density evolution for this type of channel can be simplified to tracking the probability distribution of the message cardinalities, effectively a $q$-ary probability vector instead of the $2^{q}$ probability vector that would be required to operate density evolution on the full message alphabet. We have shown some preliminary numerical results, listing thresholds that emerge from the density evolution recursion, alongside a conjectured estimate for the rate of long SUDOKU-type codes.

Furter work will require a firmer grasp on the code rate and comparison of the thresholds for other code dimensions. Also, a simulation of code performance for various block lengths would be of interest but full implementation of an encoder and decoder for SUDOKU-type codes still requires solutions of some unsolved technical details, as mentioned in the introduction.

\section{REFERENCES}

[1] P. Farrell, "Sudoku codes: a tutorial," in Proc. Int. Symp. on Commun. Theory and App. (ISCTA), Ambleside, U.K., Jul. 2009.

[2] T. K. Moon and J. H. Gunther, "Multiple constraint satisfaction by belief propagation: An example using sudoku," in Proc. SMC Conference on Automated Learning Systems, Jul. 2006, pp. 122-126.

[3] T. K. Moon, J. H. Gunther, and J. J. Kupin, "Sinkhorn solves sudoku," IEEE Trans. Inf. Theory, vol. 55, no. 4, pp. 1741 - 1746, Apr. 2009.

[4] T. J. Richardson and R. L. Urbanke, "The capacity of low-density paritycheck codes under message-passing decoding," IEEE Trans. Inf. Theory, no. 2, pp. 599-618, Feb. 2001. 